\title{
ACTH and cortisol secretion in patients with Alzheimer's disease
}

\author{
Massimo Franceschi, Lorena Airaghi, Cristina Gramigna, Giulio Truci, \\ Maria Grazia Manfredi, Nicola Canal, Anna Catania
}

\begin{abstract}
The "glucocorticoid cascade hypothesis" for pathological ageing of the brain is supported by strong experimental data, but the clinical correlates are far less clear. The basal ACTH and cortisol secretion have been studied before and after the dexamethasone suppression test in patients in the early stages of clinically probable Alzheimer's disease and in controls, and the results were all normal. These findings do not support the hypothesis that the pathological brain ageing of Alzheimer's type is caused by hyperactivity of the pituitaryadrenal axis.
\end{abstract}

Alzheimer's disease (AD) is the most prevalent degenerative disorder causing dementia. The possible causes of $\mathrm{AD}$ are currently being extensively investigated, particularly the roles of heredity, viruses, environmental toxic agents and immunological factors. The aetiology of the disease, however, is still unknown. One of the less frequently investigated, though challenging, hypotheses for pathological brain ageing, is the "glucocorticoid cascade hypothesis", based on evidence that increased secretion of glucocorticoids and/or prolonged exposure to hypercortisolism can damage the hippocampal neurons. ${ }^{23}$

The hippocampus is the site of early pathological lesions of $\mathrm{AD}$. This brain region, critical for memory performance, mediates the inhibition of glucocorticoid secretion arising at the end of the stress. ${ }^{4}$ Since hyperactivity of the hypothalamic-pituitary-adrenal (HPA) axis has been described in $A D$ patients, ${ }^{5-8}$ hippocampal degeneration has been proposed as either the cause or the effect of HPA hyperactivity.

Adrenal hyperactivity in $\mathrm{AD}$ patients is not universally accepted, however, and normal activity of HPA axis has been reported. . $^{-11}$ Our study was performed to evaluate the clinical equivalent of the "glucocorticoid cascade hypothesis" in view of the conflicting results of previous studies. Dexamethasone suppressibility of plasma ACTH and cortisol and the circadian rhythm of cortisol in patients with mild to moderate $\mathrm{AD}$ were compared with those of normal controls.

\section{Subjects and methods}

Fourteen patients with $\mathrm{AD}, 10$ women and four men, aged 59-79 years, were studied. All had clinically probable $\mathrm{AD}$, according to standard research criteria. ${ }^{12}$ They had had mild to moderate dementia for two to four years.

The controls were 13 age matched subjects, four women and nine men, with normal neurological examination and history, admitted to hospital for minor medical problems. Patients and controls had been admitted to hospital for at least one week and were free of drugs when tested. The controls and 10 patients gave their consent to the study; permission was given by the next of kin for the other four patients.

Exclusion criteria were: history or clinical evidence of depression, as evaluated by the Hamilton Depression Scale; obesity and endocrinopathy; infectious and neoplastic disorders.

After an overnight fast, a blood specimen was taken at 8.00 am for basal hormone determinations. Blood samples for ACTH determination were collected in siliconised tubes containing EDTA and aprotinin, immediately centrifuged and stored in aliquots at $-20^{\circ} \mathrm{C}$. Blood samples for cortisol determination were collected in heparinised tubes, aliquoted and stored at $-20^{\circ} \mathrm{C}$

An overnight suppression test was performed administering $1 \mathrm{mg}$ of dexamethasone IM, at $11.00 \mathrm{pm}$. Blood samples were collected at $8.00 \mathrm{am}$ and $4.00 \mathrm{pm}$ the following day (normal inhibition of plasma cortisol $<7 \mu \mathrm{g} / \mathrm{dl}) .^{13}$

Circadian rhythm of cortisol was evaluated at 8.00 am (normal range $6-23 \mu \mathrm{g} / \mathrm{dl}$ ) and $11.00 \mathrm{pm}$ (normal $<7 \mu \mathrm{g} / \mathrm{dl}$ ), in seven patients, two men and five women.

Plasma ACTH was measured by an IRMA method (Nichols Institute, San Juan Capistrano, CA); normal range 9-52 pg/ml; intra-assay $\mathrm{CV} 3.0 \%$; inter-assay CV $7.8 \%$; sensitivity $1 \mathrm{pg} / \mathrm{ml}$. Plasma cortisol was measured in unextracted plasma by solid phase RIA (Sorin, Saluggia, Italy). 


\section{Results}

Basal plasma ACTH and cortisol concentration, mean $(\mathrm{SD})$, were similar in $\mathrm{AD}$ patients [23.4 (3.3) pg ACTH $/ \mathrm{ml}$ and 15.5 (5) $\mu \mathrm{g}$ cortisol $/ \mathrm{dl}]$ and controls $[21.9(9.3) \mathrm{pg} / \mathrm{ml}$ and $14 \cdot 8(6 \cdot 1) \mu \mathrm{g} / \mathrm{dl}]$.

After dexamethasone administration, normal inhibition of plasma cortisol was observed in $13 / 14$ patients and in $12 / 13$ controls. Cortisol levels in the $\mathrm{AD}$ group were $1 \cdot 2(1 \cdot 1)$ [versus $2.0(1.3)$ in controls] at 8.00 am and $3.0(5.3)$ [versus $2.4(1.6)$ in controls] at $4.00 \mathrm{pm}$.

After dexamethasone administration, both patients and controls showed significant reductions of ACTH plasma concentrations: $8 \cdot 4$ $(2 \cdot 1)$ versus $7 \cdot 5(1 \cdot 5)$ at 8.00 am and $9 \cdot 2(6 \cdot 2)$ versus $7 \cdot 8(1 \cdot 7)$. The degree of $\mathrm{ACTH}$ reduction was similar for the two groups.

The circadian rhythms of plasma cortisol were normal for the seven $A D$ patients in whom it was studied: $16.6(3.3)$ at $8.00 \mathrm{am}$, and 3.9 $(1.2)$ at $11 \mathrm{pm}$. There was no correlation in the $\mathrm{AD}$ patients between severity of dementia or duration of the disease and basal and postdexamethasone levels of ACTH or cortisol.

\section{Discussion}

This study of $\mathrm{AD}$ patients in early stages of their disease demonstrated normal secretory dynamics of circulating ACTH and cortisol.

The dexamethasone suppression test has been widely used to study demented patients, ${ }^{5-11}$ mainly to differentiate organic dementia from depressive pseudodementia. ${ }^{58}$ Conflicting results have been reported and this test is considered to be of limited usefulness for diagnostic purposes. ${ }^{78}$

The prevalence of non-suppressor demented patients after dexamethasone administration have differed: $7 / 15,{ }^{5} 12 / 21,{ }^{6} 3 / 18,{ }^{7}$ or $7 / 27 .^{8}$ The probability of being a non-suppressor correlated sometimes with age, ${ }^{8}$ sometimes with severity of dementia, ${ }^{68}$ sometimes with no clinical features. ${ }^{9}$ However, in agreement with this study, normal responses to dexamethasone inhibition in all the patients have also been reported by others. ${ }^{9-11}$

Increased basal plasma cortisol was found in demented patients ${ }^{11}$ and Raskind et $a l^{5}$ also reported blunted cortisol circadian rhythms in non-suppressor demented patients. In seven of our 14 patients, all inhibited by dexamethasone, we found normal circadian cortisol rhythm, supporting previous reports. ${ }^{810}$

The discrepancies among the results of various studies may have different explanations. First, earlier studies may have suffered from methodological bias due to inadequate diagnostic criteria for differentiation of primary dementia of Alzheimer's type from other types of dementias.

Moreover, some of these studies did not adequately describe the clinical features of the patients investigated. Patients included in previous studies also differed widely in age, duration and severity of disease, all parameters reported to influence the neuroendocrine profile. Finally, other methodological differences in these studies may be important: inpatient or outpatient, control groups, concomitant therapy, affective or clinical disorders. This study which supports previous research, excludes adrenal hyperactivity or HPA dysfunction as a general feature of $\mathrm{AD}$ at least in its early stage.

1 Sapolsky RM, Krey LC, McEwen DS. The neuroendocrinology of stress and aging: the glucocorticoid cascade crinology of stress and aging: the glucocor

2 Landfield P, Waymire J, Lynch G. Hippocampal aging and adrenocorticoids: a quantitative correlation. Science 1978; 202:1098.

3 Sapolsky RM, Pulsinelli W. Glucocorticoids potentiate ischemic injury to neurons: therapeutic implications. Science 1985;229:1397.

4 Feldman S, Conforti N. Participation of the dorsal hippocampus in the glucocorticoid feedback effect on adrenocortical activity. Neuroendocrinology 1980;30:52.

5 Raskind M, Peskind E, Rivard M. Dexamethasone suppression test and cortisol and circadian rhythm in primary degent and cortisol and circadian rhy 1982;139:1468.

6 Balldin J, Gottfries C, Karlsson I, Lindstedt G, Langstrom G, Walinder J. Dexamethasone suppression test and serum prolactin in dementia disorders. Br J Psychiatry 1983;143:277.

7 Georgotas A, McCue RE, Kim OM, et al. Dexamethasone suppression in dementia, depression, and normal aging. Am J Psychiatry 1986;143:452-6.

8 Davous P, Roudier M, Piketty ML, Abramowitz C, Lamour Y. Pharmacological modulation of cortisol secretion and dexamethasone suppression in Alzheimer's disease. Biol Psychiatry 1988;23:13-24.

9 Carnes M, Smith JC, Kalin N, Bauwens SF. Effects of chronic medical illness and dementia on the dexamethasone suppression test. J Am Geriatr Soc 1983;31:269-71.

10 Pomara N, Oxenkrug GF, McIntyre IM, Block R, Stanley $M$, Gerson S. Does severity of dementia modulate response to dexamethasone in individuals with primary sponse to dexamethasone in individuals with primary

11 Charles GA, Lefevre A, Mirel J, Rush AJ, Suppression of cortisol following dexamethasone suppression test in depression and other psychiatric illness. Br J Psychiatry depression and other

12 McKhann G, Drachman D, Folstein M, Katzman R, Price D, Stadlan EM. Clinical diagnosis of AD: report of the NINCDS-ADRDA work group under the auspices of Health and Human Service Task Force on AD. Neurology 1984;34:939.

13 Jacobs HS, Nabarro JDN. Tests of hypothalamic-pituitaryadrenal function in man. QJ Med 1969;38:475-80. 